\title{
Phase recovery by using optical fiber dispersion and pulse pre-stretching
}

\author{
C. Cuadrado-Laborde $\cdot$ M. Brotons-Gisbert • \\ G. Serafino $\cdot$ A. Bogoni $\cdot$ P. Pérez-Millán • \\ M. V. Andrés
}

Received: 13 June 2014/ Accepted: 29 September 2014/Published online: 12 October 2014

(C) Springer-Verlag Berlin Heidelberg 2014

\begin{abstract}
In this work, the applicability of a recently proposed phase recovery technique is extended, by using temporal pulse pre-stretching. As a proof of concept, the light pulses emitted by a two-stage laser system consisting of a sub-picosecond passively mode-locked fiber laser followed by an erbium-doped fiber amplifier were experimentally analyzed. These results were successfully compared with two indirect phase recovery techniques, both based on the Gerchberg-Saxton recursive algorithm and with the intensity autocorrelation when sub-picosecond light pulses were analyzed.
\end{abstract}

C. Cuadrado-Laborde $(\bowtie) \cdot$ M. Brotons-Gisbert .

P. Pérez-Millán · M. V. Andrés

Departamento de Física Aplicada y Electromagnetismo,

ICMUV, Universidad de Valencia, C/Dr. Moliner 50,

46100 Burjassot, Valencia, Spain

e-mail: Christian.Cuadrado@uv.es

C. Cuadrado-Laborde

Instituto de Física Rosario (CONICET-UNR), Blvr. 27 de

Febrero 210bis, S2000EZO Rosario, Argentina

G. Serafino

TeCIP Institute, Scuola Superiore Sant'Anna, Via Moruzzi 1, 56124 Pisa, Italy

\footnotetext{
A. Bogoni

National Laboratory of Photonic Networks, Inter-university

National Consortium for Telecommunications (CNIT), Via

Moruzzi 1, 56124 Pisa, Italy

P. Pérez-Millán

FYLA LASER SL, Ronda Guglielmo Marconi 12,

46980 Valencia, Spain
}

\section{Introduction}

The phase recovery belongs to the class of inverse problems whose resolution can be sought through either direct or indirect methods [1]. In the former, the sought solution is expressed through one or several equations explicitly as a function of the input data; its numerical efficiency is high, and its non-iterativity turns it very fast. Unlike direct methods, which attempt to calculate an exact solution in a finite number of operations, indirect methods starts with an initial approximation (generally an educated guess) and generates successively improved approximations in an infinite sequence whose limit is the exact solution. As a drawback, indirect methods are often iterative, and therefore, computationally intensive, being slower than their direct counterpart. A well-known example of indirect method, within the phase recovery framework, is given by the Gerchberg-Saxton (GS) algorithm and further modifications $[2,3]$. The transport of intensity equation (TIE), on the other hand, belongs to the direct methods for phase recovery [4-6].

In fiber-optic communication systems today is of great importance the phase monitoring of the transmitted light pulses, since it influences strongly the overall system's performance. Recently, three different direct methods for phase retrieval were proposed, especially well suited to analyze light pulses in the range from a few picoseconds to nanoseconds regime [7-10]. Reference [7] showed that a spectrally shifted differentiator can be used to retrieve the phase profile of a given temporal optical waveform. However, the operation is performed by using short- or long-period fiber gratings, which have fixed operation wavelengths and restrictive operation bandwidths. In [8, 9], a direct phase recovery technique was proposed from temporal intensity measurements at the input and output of 
a linear optical filter. However, precise knowledge of the filter's impulse response is necessary in amplitude and phase, together with the additional restriction in the later to be limited to a maximum variation range of $\pi$ rad through the whole operation bandwidth. Very recently, a direct method for phase recovery based on the use of the TIE was introduced, which combines a first experimental stage followed by a one-step numerical stage [10]. In the experimental stage, two temporal intensity profiles at the input and output of a linear dispersive device are acquired with an oscilloscope, whereas in the numerical stage, a single equation is applied to retrieve the phase profile in just one step. The proposal belongs to the framework of the use of dispersive elements to characterize optical signals by using oscilloscope measurements, such as the well-known dispersive Fourier transformation (DFT) [11]. In this work, the applicability of the direct phase recovery technique proposed in Ref. [10] is extended by using temporal pulse pre-stretching. As an example, pulse pre-stretching can be necessary because of insufficient bandwidth of the available oscilloscope. This scenario was experimentally tested by analyzing the light pulses through a two-stage laser system consisting of a sub-picosecond passively modelocked fiber laser followed by an erbium-doped fiber amplifier (EDFA). These results were compared with two indirect phase recovery techniques, both based on the GS recursive algorithm. Finally, the auto-correlation obtained from the recovered temporal intensity profiles is compared with the experimental measurement of the intensity autocorrelation, when sub-picosecond light pulses were analyzed.

\section{Theory}

In this section, the theory behind the phase recovery methods used in this work is reviewed. These phase recovery methods require as input data one of the following:

- Two different temporal intensity profiles

- One temporal intensity profile and one optical power spectrum.

It is worth to emphasize that other approaches based only on the use of the optical power spectrum and intensity autocorrelation, i.e., via the Wiener-Khinchin theorem, have demonstrated to give ambiguous solutions for the phase retrieval problem [12]. Therefore, its use is not an acceptable option. In the following, we start with the Fresnel transform (FrT) phase recovery technique, following with a variation of the GS recursive algorithm, and finally introducing the original GS algorithm.

\subsection{Fresnel transform phase recovery}

The phase of a given one-dimensional complex signal $f(t)=|f(t)| \exp [j \varphi(t)]$ can be simply expressed as a function of its instantaneous angular frequency $\varphi^{\prime}(t)=\mathrm{d} \varphi(t) / \mathrm{d} t$, through:

$\varphi(t)=\varphi_{0}+\int_{-\infty}^{t} \mathrm{~d} \tau \varphi^{\prime}(\tau)$,

where $\varphi_{0}$ is an arbitrary phase constant. The TIE equation provides the link to recover the instantaneous angular frequency $\varphi^{\prime}(t)$, provided the transversal derivative of the FrT of $f(t)$ is known, through [13]:

$\varphi^{\prime}(t)=\left.\frac{1}{|f(t)|^{2}} \int_{-\infty}^{t} \frac{\partial\left|f_{\alpha}(\tau)\right|^{2}}{\partial \alpha}\right|_{\alpha=0} \mathrm{~d} \tau$,

where $f_{\alpha}(t)$ is the FrT of $f(t)$, and $\alpha$ its FrT parameter. As far as we know, the first time the TIE was used in the temporal domain was in Ref. [14], when it was applied to determine the nonlinear coefficients of a highly nonlinear fiber. Regarding the FrT of a given signal, it can be performed optically by propagating the input optical pulse $f(t)$ by an optical fiber, whose transfer function $S(\omega)$ can be approximated, within a certain operative spectral bandwidth, as:

$S(\omega)=\exp \left(-j \Phi_{20} \omega^{2} / 2\right)$,

where $\omega$ is the baseband angular frequency. In that case, the FrT parameter $\alpha=\Phi_{20}$, where $\Phi_{20}$ is the first-order dispersion coefficient of the optical fiber. In turn, $\Phi_{20}$ can be related with the second-order derivative of the propagation constant $\beta_{20}$ of the optical fiber through $\Phi_{20}=L \beta_{20}$, where $L$ is the optical fiber length. Regarding the derivative of the FrT signal intensity with respect to the FrT parameter, see Eq. (2), it can be replaced by a finite difference approximation:

$\left.\left.\frac{\partial\left|f_{\alpha}(t)\right|^{2}}{\partial \alpha}\right|_{\alpha=0} \approx \frac{\left|f_{\alpha}(t)\right|^{2}-|f(t)|^{2}}{\alpha}\right|_{\alpha \rightarrow 0} ;$

where the numerator of the right-hand side of Eq. (4) is known as the temporal profile difference (TPD). The validity of Eq. (2) is restricted to the near-field regime, where the fiber length fulfills the following inequality:

$L_{\mathrm{NF}} \ll \frac{\Delta t^{2}}{2 \pi\left|\beta_{20}\right|}$,

where $L_{\mathrm{NF}}$ is the near-field length. However, it is important to keep in mind that enough fiber length is still required to get enough TPD, in order to minimize the noise influence. Finally, it is worth mentioning that Eq. (2) has a spectral counterpart, which was applied to characterize the electric 
field of a light pulse by performing two different spectral measurements after a quadratic phase modulation [15].

\subsection{Fresnel transform Gerchberg-Saxton algorithm}

The GS algorithm was originally developed to retrieve the phase of a spatial image based on intensity recordings in the image and diffraction planes [2]. The method depends on there being a Fourier transform relation between the waves in these two planes. However, an adaptation of this concept is suitable for the present problem, replacing the Fourier transform (FT) by a FrT [16], relaxing in this way the use of high dispersion values. This phase retrieval technique also requires the measurement of the temporal intensity profiles of the original $|f(t)|^{2}$ and FrT $\left|f_{\alpha}(t)\right|^{2}$ signals, i.e., at the input and output of an optical fiber of known dispersion. The proposed algorithm is illustrated schematically in Fig. 1, which should be followed clockwise. To begin the procedure (upper left corner), an educated guess for $\varphi(t)$ is required, which is necessary only for the first roundtrip. The educated phase guess and measured magnitude $|f(t)|$ are combined and numerically Fourier transformed. Once in the frequency domain, the dispersion contribution $S(\omega)$ is applied [see Eq. (3)], and the waveform is transformed back to time. At this point, only the calculated phase $\phi(t)$ is retained; the calculated FrT magnitude $\left|f_{\alpha}^{\prime}(t)\right|$ is replaced by the measured FrT magnitude $\left|f_{\alpha}(t)\right|$. The calculated phase and measured FrT magnitude are combined and numerically Fourier transformed. Once in the frequency domain, the dispersion contribution is removed by using $S^{-1}(\omega)$, and the waveform is transformed back to time. The cycle is then completed by retaining only the calculated phase $\varphi(t)$, whereas the calculated magnitude $\left|f^{\prime}(t)\right|$ is replaced by the measured magnitude $|f(t)|$. This iterative algorithm can be executed as many times as required.

\subsection{Gerchberg-Saxton algorithm}

This technique is the time-domain version of the classical GS algorithm [2]. This phase retrieval technique requires

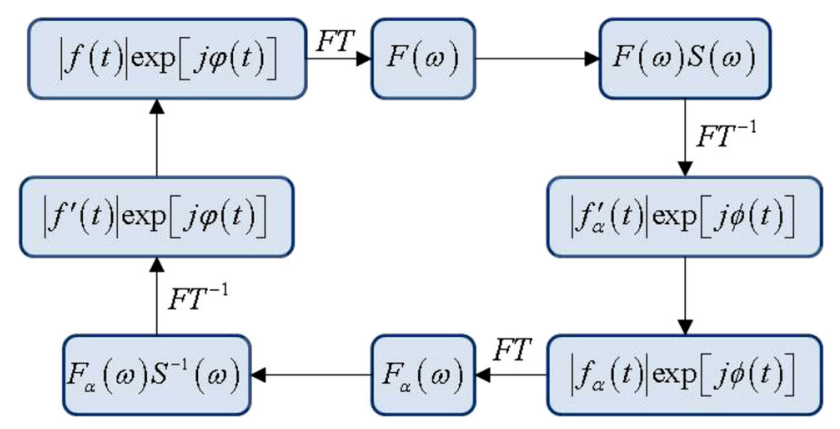

Fig. 1 FrT-GS algorithm for temporal phase retrieval one temporal intensity profile $|f(t)|^{2}$ and one optical power spectrum $|F(\omega)|^{2}$. The algorithm goes back and forth between these experimentally measured intensities, very similarly as described in Sect. 2.2. The proposed algorithm is illustrated schematically in Fig. 2, which should be followed clockwise, beginning by the upper left corner. As opposed to techniques described before in Sects. 2.1 and 2.2 , a dispersive media is not a priori necessary, i.e., both measurements are performed in the same point.

The optical power spectrum $|F(\omega)|^{2}$ can be obtained in two different ways, either by using an optical spectrum analyzer (OSA) or by DFT. In the former, the inherent single-shot capabilities of this phase retrieval technique are inhibited, whereas in the later are preserved. This distinction could be important, e.g., when it is necessary to recover the phase of a non-periodical signal. In the DFT, the optical signal is propagated long enough that the Fraunhofer approximation holds $\Delta t^{2} / 2 \pi\left|\Phi_{20}\right| \ll 1$ (far-field regime). In that case, the transmitted signal envelope is, within a phase factor, proportional to the FT of the input signal envelope [17]. In this case, a second temporal intensity profile is registered, which represents the signal spectrum in intensity. In the downside, it is generally necessary to use high dispersion values, which translates in the use of a long length of optical fiber.

\subsection{The pre-stretching technique}

Techniques described in Sects. 2.1-2.3 require at least one temporal intensity profile of the signal under test, i.e., the true intensity profile of the envelope of the optical field. Therefore, these techniques are limited by the typical bandwidth of temporal test equipment, i.e., the sampling scope of the available oscilloscope. In particular, a $50 \mathrm{GHz}$ bandwidth oscilloscope can perform the detection of arbitrary light pulses ranging from $\sim 10 \mathrm{ps}$ of temporal width, and a much more expensive $100 \mathrm{GHz}$ bandwidth oscilloscope can extend this detection range up to $\sim 5 \mathrm{ps}$ of temporal width. Thus, these techniques could be directly applied only over optical pulses with time features $>5$ ps or $>10 \mathrm{ps,}$ depending of the available oscilloscope. To overcome this limitation, we propose here to use the pre-stretching technique, by using a well-characterized

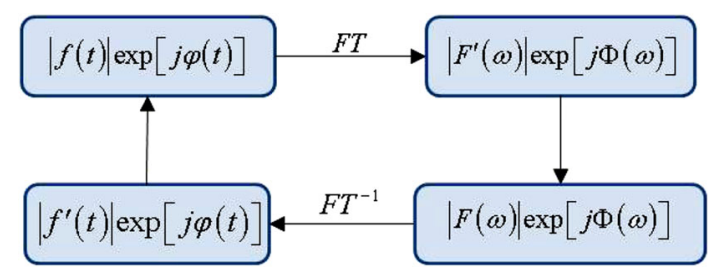

Fig. 2 GS algorithm for temporal phase retrieval 
dispersive optical fiber, in order to temporally stretch the optical pulse under test before the application of the proposed techniques [18]. Using this simple linear method, it is possible to characterize shorter light pulses. For ultrashort pulses (temporal width $<1 \mathrm{ps}$ ), nonlinear effects become increasingly important, being Eq. (3) unsuitable to describe the increasingly complex behavior of the optical fiber, establishing in this way a lower limit for the prestretching technique.

The short pulse $f_{0}(t)=\left|f_{0}(t)\right| \exp \left[j \varphi_{0}(t)\right]$ is propagated through a dispersive media-whose transfer function is known, let us denote it by $S_{0}(\omega)$ - until it temporally broadens enough that its measurement by an oscilloscope is possible. Let us denote this pre-stretched pulse by $f(t)=|f(t)| \exp [j \varphi(t)]$. Next, techniques 2.1-2.3 can be applied in the usual way, and the phase $\varphi(t)$ of the prestretched pulse is recovered. Now, since $f(t)$ is known in both amplitude and phase, the numerical FT of $f(t)$ can be calculated, let us denote it by $F(\omega)$. The short pulse $f_{0-}$ ( $t$ ) can be recovered in both amplitude and phase, by removing the contribution of the pre-stretching dispersion, $S_{0}(\omega)$, to the spectrum of $f(t)$, followed by an inverse FT of the resulting spectrum, in the following way:

$f_{0}(t)=\left|f_{0}(t)\right| \exp \left[j \varphi_{0}(t)\right]=\mathrm{FT}^{-1}\left\{\frac{F(\omega)}{S_{0}(\omega)}\right\}$.

High-order dispersion terms in $S_{0}(\omega)$, which may be needed for wide spectral windows, can be easily included.

\section{Experiment}

As a proof of concept, the light pulses of a laser system consisting of a passively mode-locked laser, whose pulses were subsequently amplified through an EDFA, were experimentally analyzed. Since the light pulses in these two stages have very different characteristics, in the following we will analyze it separately.

\subsection{Characterization of the light pulses at the output of the mode-locked laser}

The available oscilloscope (100 GHz bandwidth) can perform the detection of arbitrary light pulses ranging from $\sim 5 \mathrm{ps}$ of temporal width. However, according to the intensity autocorrelation measurements, the output of the mode-locked laser is in the sub-picosecond regime; therefore, it will be necessary to use the pre-stretching technique. As a consequence, there are two dispersive lines of length $L_{0}$ and $L_{1}$, being the former for pre-stretching and the later for the phase recovery technique, see Fig. 3. The light pulses were measured in every stage by using an

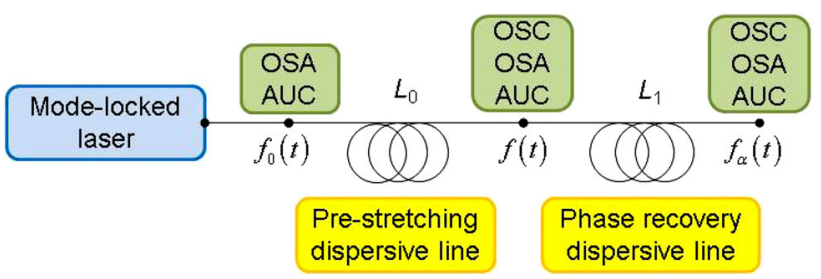

Fig. 3 Experimental setup for phase recovery of the output light pulses of the mode-locked laser, in the presence of pulse prestretching

oscilloscope, OSA, and autocorrelator, except immediately after the mode-locking laser, where oscilloscope measurements could not be performed. The oscilloscope was triggered by using the same light pulse via a photodetector.

The passively mode-locked fiber laser provided light pulses at an emission wavelength $\lambda_{0}=1,556.87 \mathrm{~nm}$ with a repetition rate of $36.77 \mathrm{MHz}$. In this stage, the temporal width of the autocorrelator profile was $\Delta t_{\mathrm{AUC}}=0.83 \mathrm{ps}$ (FWHM). As dispersion line, a standard telecom optical fiber (SMF-28 by Corning) was used. Since the spectral bandwidth of the light pulses under study is nonnegligible, the variation of the first-order dispersion $D$ as a function of the optical wavelength was taken into account, which can be approximated by $D(\lambda)=S_{\mathrm{ZD}}\left(\lambda-\lambda_{\mathrm{ZD}}^{4} / \lambda^{3}\right) / 4$, where $\lambda_{\mathrm{ZD}}=1,313 \mathrm{~nm}$ is the zero-dispersion wavelength, and $S_{\mathrm{ZD}}=0.086 \mathrm{ps} /\left(\mathrm{nm}^{2} \mathrm{~km}\right)$ is the zero-dispersion slope. The inclusion of a third-order dispersion term was not necessary, since the central wavelength of the pulse under analysis is not close to $\lambda_{Z D}$. The length of the pre-stretching line was selected as $L_{0}=92.5 \mathrm{~m}$, which was enough to pre-stretch the sub-picosecond light pulses to a ten-of-ps regime, where oscilloscope measurements can be performed. At the end of the pre-stretching dispersive line, the light pulses have a temporal width $\Delta t=9 \mathrm{ps}$ (FWHM) and a peak power $P_{0}=0.4 \mathrm{~W}$, which was derived using a measured average power of $0.15 \mathrm{~mW}$. The near-field length—calculated by using Eq. (5) with $\beta_{20}\left(\lambda_{0}\right)=\lambda_{0}^{2}$ $D\left(\lambda_{0}\right) / 2 \pi \mathrm{c}=-20 \mathrm{ps}^{2} / \mathrm{km}$-results in $L_{\mathrm{NF}} \ll 586 \mathrm{~m}$. As a result, for the phase recovery dispersive line a dispersion length $L_{1}=25 \mathrm{~m}$ was used, which fulfills the near-field requirement. On the other hand, the nonlinear length for the phase recovery line $L_{\mathrm{NL}}$ was obtained from $L_{\mathrm{NL}}=$ $\left(\gamma P_{0}\right)^{-1}=2 \mathrm{~km}$, by using a nonlinear parameter $\gamma \approx 0.001 \mathrm{~W}^{-1} / \mathrm{m}$ (derived using a mode field diameter of $10.4 \mu \mathrm{m}$ and a nonlinear index coefficient $n_{2}=2.6 \times$ $\left.10^{-20} \mathrm{~m}^{2} / \mathrm{W}\right)$. In the following, since $L_{1}<<L_{\mathrm{NL}}$, the influence of nonlinear effects in the phase recovery dispersive line can be safely ignored.

Figure 4a shows the measured temporal intensity profiles of the pre-stretched and FrT pulses, i.e., $|f(t)|^{2}$ and 

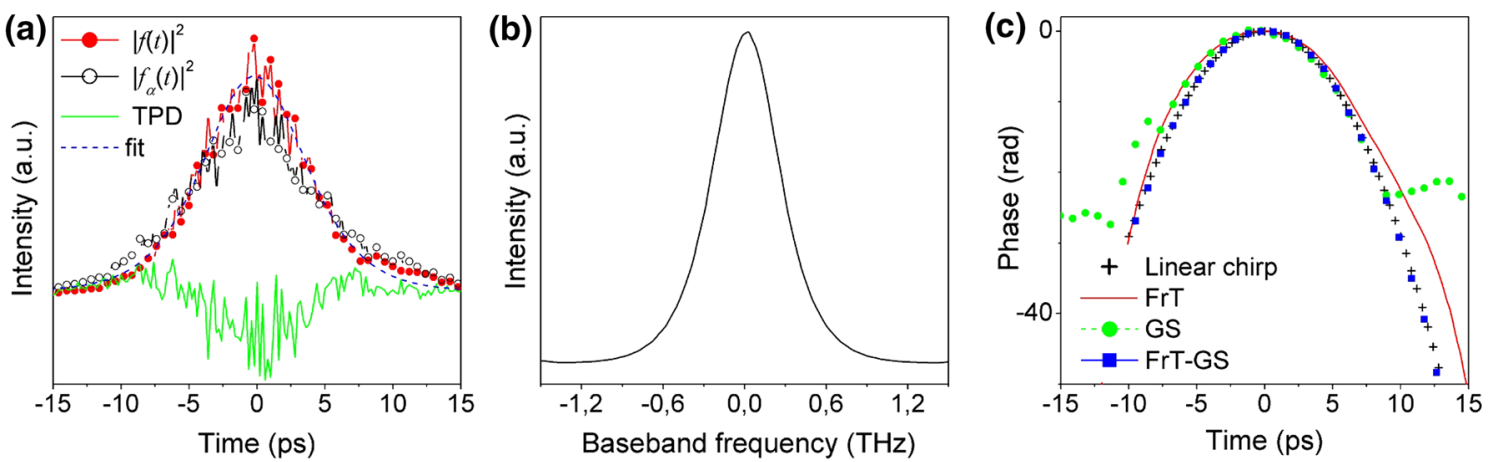

Fig. 4 a Measured temporal profiles at the input and output of the phase recovery dispersive line, i.e., $|f(t)|^{2}$ and $\left|f_{\alpha}(t)\right|^{2}$, respectively; the TPD and fitting of $|f(t)|^{2}$ are also shown. b Optical power spectrum measured at the input of the phase recovery dispersive line.

$\left|f_{\alpha}(t)\right|^{2}$, together with its TPD. The noise content in the TPD was mainly due to a sequential measurement of $|f(t)|^{2}$ and $\left|f_{\alpha}(t)\right|^{2}$. The measurement sensitivity was therefore limited because of the laser amplitude noise and thermal and mechanical drift of the laser and measurement setup [19]. However, the noise content in the TPD could be highly reduced with a simultaneous measurement of $|f(t)|^{2}$ and $\left|f_{\alpha}(t)\right|^{2}$. It is worth to emphasize that this measurement simultaneity is perfectly allowed by our experimental setup, provided the available oscilloscope has two independent channel operatives for this task. Figure $4 \mathrm{~b}$ shows the measured spectrum as a function of the baseband optical frequency, the spectral linewidth $\Delta v=726 \mathrm{GHz}$ (FWHM) $(\Delta \lambda=5.9 \mathrm{~nm})$. The phase recovered for $f(t)$ by using the technique described in Sect. 2.1 is shown in Fig. 4c. This phase profile was directly recovered from the raw data, being unnecessary to smooth previously the data set. The instantaneous angular frequency-obtained from Eq. (2), not shown-decreases monotonously from the leading to the trailing edge of the pulse, which is currently known as a down-chirp. For comparison purposes, the prestretched pulse $f(t)$ can be approximately fitted in both, modulus and phase, through a linearly chirped secant hyperbolic profile $f(t)=\operatorname{sech}\left(t / T_{0}\right) \times \exp \left(-j C t^{2} / 2 T_{0}^{2}\right)$, with $T_{0}=5.1 \mathrm{ps}$ and $C=15$, see Fig. $4 \mathrm{a}$, c. The phase recovered by using the FrT-GS technique described in Sect. 2.2 is also shown in Fig. 4c, after 60 roundtrips. The educated phase guess was a parabolic profile, which is a reasonable choice since $f(t)$ has been linearly chirped by the pre-stretching dispersion line. In Fig. 4c, it is also shown the phase recovered after 60 roundtrips by using the GS technique described in Sect. 2.3, by using the temporal intensity profile and the optical power spectrum at the end of the pre-stretching line, shown in Fig. 4a, b, respectively. The same educated phase guess used for the FrT-GS phase recovery technique of Sect. 2.2 was used. The phases recovered match with reasonable accuracy, although it c Temporal phase profiles experimentally recovered at the input of the phase recovery dispersive line by the FrT, FrT-GS, and GS phase recovery techniques; a parabolic phase profile with linear chirp parameter $C=15$ is also shown

should be mentioned that in the FrT technique, it is not necessary an educated guess for the phase.

Once the phase of the pulse at the output of the prestretching line is known, it is possible to apply the prestretching technique to fully recover the pulse at the output of the mode-locked laser, see Fig. 3. It is important to note that the model of a purely dispersive transfer function for the pre-stretching dispersive line is justified only when the peak power of the transmitted light pulse is moderately low. This can be checked by calculating the nonlinear length for the pre-stretching line, which results in $L_{\mathrm{NL}}=\left(\gamma P_{0}\right)^{-1}=122 \mathrm{~m}$, where now $P_{0}=6.6 \mathrm{~W}$ is the peak power at the input of the pre-stretching line, derived using a measured average power of $0.15 \mathrm{~mW}$ and assuming a temporal width $0.65 \times \Delta t_{\mathrm{AUC}}=0.65 \times 0.83 \mathrm{ps}=$ 0.54 ps. Since the selected pre-stretching line length $L_{0}=92.5 \mathrm{~m}$ is below the $L_{\mathrm{NL}}$, in the following the influence of nonlinear effects can be ignored. Once the Fourier transform of the pre-stretched pulse is numerically obtained by using the intensity and phase profiles shown in Fig. 4a, c, respectively, Eq. (6) can be used to obtain the pulse in both amplitude and phase at the output of the mode-locked laser $f_{0}(t)$. In Fig. 5a, it is shown the intensity of $f_{0}(t)$ recovered by using the different techniques. Regarding the recovered phases, see Fig. 5b, each one of the techniques shows an approximately linear phase, which indicates the absence of frequency chirp in the output light pulses of the mode-locked laser. It should be emphasized that our attention should be focused in the region where the pulse energy is concentrated, i.e., from -0.5 to $0.5 \mathrm{ps}$, being the rest of the phase profile meaningless. It is worth to emphasize that the characterization of the pre-stretching dispersive line is critical when ultra-short pulses are under analysis, as it was previously demonstrated in Ref. [20]. As an additional confirmation of the recovered temporal intensity profile for $f_{0}(t)$, Fig. $5 \mathrm{c}$ shows the autocorrelation curve calculated from the recovered temporal pulse 


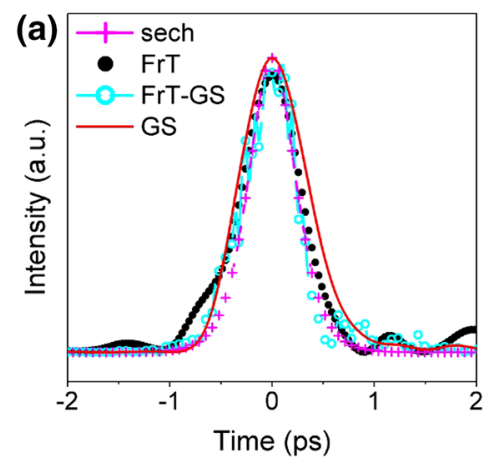

Fig. 5 Temporal intensity and phase profiles of the output light pulses of the mode-locked laser, experimentally recovered by the FrT, FrT-GS, and GS phase recovery techniques, (a) and (b), respectively.

intensity profiles shown in Fig. 5a and that directly measured with a commercial auto-correlator. For secant hyperbolic-shaped pulses, the pulse duration is $\approx 0.65$ times the temporal width of the measured auto-correlation signal $\Delta t_{\mathrm{AUC}}$. Since $\Delta t_{\mathrm{AUC}}=0.83 \mathrm{ps}$, the corresponding secant hyperbolic pulse would be $0.54 \mathrm{ps}$, which was also shown in Fig. 5a, labeled as "sech" profile for comparison purposes. Finally, when another pre-stretching dispersion line length was used-i.e., $L_{0}=117.5 \mathrm{~m}$, and keeping constant the phase recovery dispersion line length, i.e., $L_{1}=25 \mathrm{~m}$-essentially the same results were obtained for the recovered intensity and phase profiles at the output of the mode-locked laser $f_{0}(t)$.

\subsection{Characterization of the light pulses at the output of the EDFA}

Our objective was to characterize the amplified light pulses $f_{0}(t)$ provided by the EDFA, see Fig. 6 . However, a certain length of optical fiber is necessary to reach the different measurement apparatus, which eventually can modify the pulse, because of the presence of dispersion. In this case, this optical fiber length-let us denote it by $L_{0}$ - can be considered as a pre-stretching dispersion line. At the end of this line, the pre-stretched pulse $f(t)$ can be measured with an oscilloscope and OSA. A second optical fiber plays the role of the phase recovery dispersive line of length $L_{1}$, at the end of which the light pulse $f_{\alpha}(t)$ can be registered with an oscilloscope and OSA. The oscilloscope was triggered by using the same light pulse via a photodetector. Therefore, once the phase of $f(t)$ is known by using the techniques discussed in Sects. 2.1-2.3; the light pulse $f_{0}(t)$ can be recovered in both amplitude and phase by applying Eq. (6).

At the output of the EDFA, the light pulses showed a broader spectrum as compared with the input, whose spectrum was shown in Fig. 4b. For this reason, it becomes

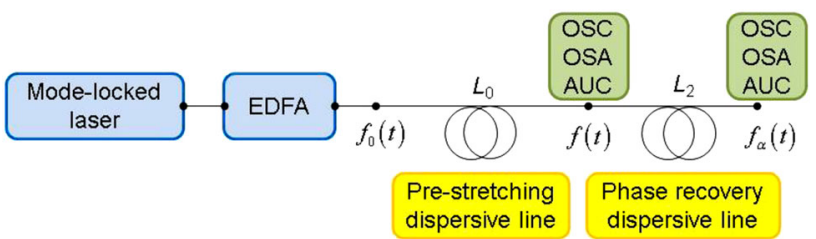

Fig. 6 Experimental setup for the phase recovery of the output light pulses of the EDFA, in the presence of pulse pre-stretching

even more relevant than before to include the change in dispersion as a function of the optical wavelength. In this case, the pre-stretching dispersive line of length $L_{0}$ was made with a SMF-28 optical fiber, whose dispersive characteristics were described before (Sect. 3.1). The phase recovery dispersive line, on the other hand, was made of a dispersion compensation fiber (DCF), whose first-order dispersion as a function of the optical wavelength can be approximated by $D(\lambda)=-113-0.44(\lambda-1,550)$, with $\lambda$ in $\mathrm{nm}$ and $D$ in $\mathrm{ps} /(\mathrm{nm} \times \mathrm{km})$. At the end of the prestretching dispersive line, the light pulses have a temporal width $\Delta t=18.42 \mathrm{ps}$ (FWHM) and a peak power $P_{0}=38 \mathrm{~W}$, which was derived using a measured average power of $29 \mathrm{~mW}$. The near-field length - calculated by using Eq. (4) with $\beta_{20}\left(\lambda_{0}\right)=-\lambda_{0}^{2} D\left(\lambda_{0}\right) / 2 \pi c=155 \mathrm{ps}^{2} /$ $\mathrm{km}$-results in $L_{\mathrm{NF}} \ll 34 \mathrm{~m}$. As a result, a dispersion length $L_{1}=2.5 \mathrm{~m}$ was used for the phase recovery dispersive line, which fulfills the near-field requirement. On the other hand, the nonlinear length for the phase recovery dispersive line $L_{\mathrm{NL}}$ was obtained from $L_{\mathrm{NL}}=\left(\gamma P_{0}\right)^{-1}=$ $22 \mathrm{~m}$, assuming a nonlinear parameter $\gamma \approx 0.001 \mathrm{~W}^{-1} / \mathrm{m}$. In the following, since $L_{1} \ll L_{\mathrm{NL}}$, the influence of nonlinear effects in the phase recovery dispersive line can be safely ignored.

Figure 7a shows the measured temporal intensity profiles of the pre-stretched and FrT pulses, i.e., $|f(t)|^{2}$ and $\left|f_{\alpha}(t)\right|^{2}$, together with its TPD, i.e., $\left|f_{\alpha}(t)\right|^{2}-|f(t)|^{2}$. 

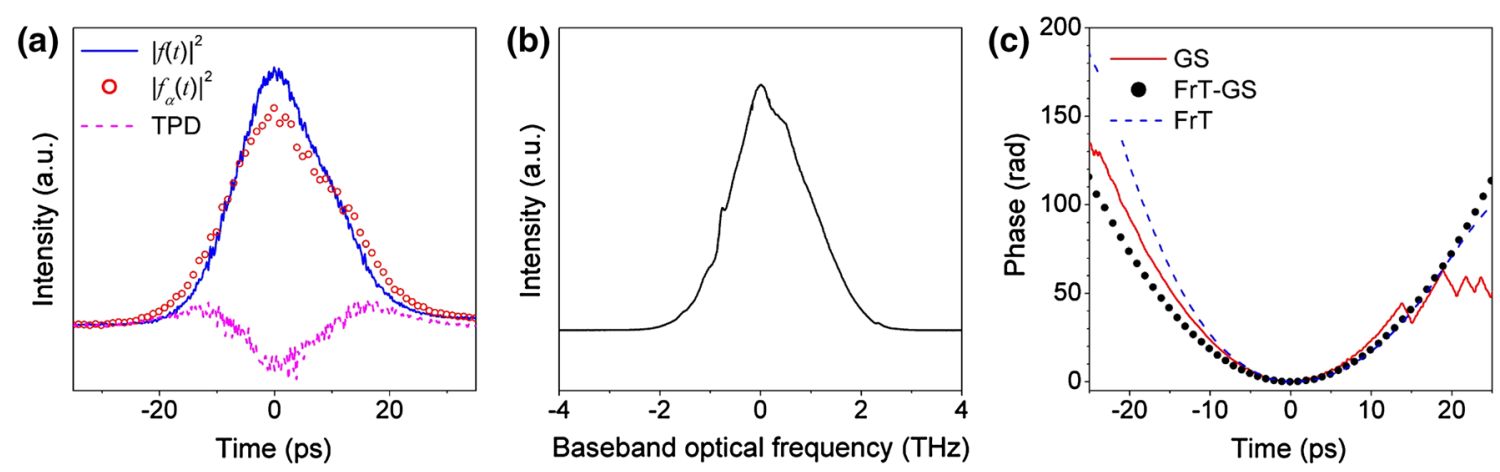

Fig. 7 a Measured temporal profiles at the input and output of the phase recovery dispersive line, i.e., $|f(t)|^{2}$ and $\left|f_{\alpha}(t)\right|^{2}$, respectively; the TPD is also shown. b Optical spectrum measured at the input of the

Figure $7 \mathrm{~b}$ shows the spectrum registered by an OSA as a function of the baseband optical frequency, the spectral linewidth $\Delta v=1.75 \mathrm{THz}$ (FWHM) $(\Delta \lambda=14.3 \mathrm{~nm})$. The phase recovered for $f(t)$ by using the technique described in Sect. 2.1 is shown in Fig. 7c. This phase profile was directly recovered from the raw data, being unnecessary to smooth previously the data set. The instantaneous angular frequency-obtained from Eq. (2), not shown-increases monotonously from the leading to the trailing edge of the pulse, which is currently known as an up-chirp. The phase recovered by using the FrT-GS technique (described in Sect. 2.2) is also shown in Fig. 7c, after 60 roundtrips. The educated phase guess was a parabolic profile. In Fig. 7c, it is shown the phase recovered after 60 roundtrips by using the GS technique (described in Sect. 2.3), by using at the end of the pre-stretching line the temporal intensity profile $f(t)$ and its corresponding intensity optical spectrum, shown in Fig. 7a, b, respectively. The same educated phase guess used for the FrT-GS technique was used here. All recovered phases match reasonably well, although it should be mentioned also that in the FrT technique, it is not necessary an educated guess for the phase.

Once the pulse at the output of the pre-stretching line $f(t)$ is known in both amplitude and phase, it is possible to take into account the pre-stretching to fully recover the pulse at the output of the EDFA, see Fig. 6. The model of a purely dispersive transfer function for the pre-stretching line is justified, since the nonlinear length $L_{\mathrm{NL}}=22 \mathrm{~m}$ is above the pre-stretching dispersive line length $L_{0}=5 \mathrm{~m}$, where the nonlinear length $L_{\mathrm{NL}}$ was assumed the same as in the phase recovery dispersive line calculated above. Once the Fourier transform of the pre-stretched pulse is numerically obtained by using the intensity and phase profiles shown in Fig. 7a, c, respectively, Eq. 6 can be used to obtain the pulse in both amplitude and phase at the output of the EDFA $f_{0}(t)$. In Fig. 8a, it is shown the intensity of $f_{0}(t)$ recovered by using the different techniques. Regarding phase recovery dispersive line. c Temporal phase profiles experimentally recovered at the input of the phase recovery dispersive line by the FrT, FrT-GS, and GS phase recovery techniques

the recovered phases, see Fig. $8 \mathrm{~b}$, the three techniques show that it is approximately parabolic, indicating the presence of a strong frequency chirp in the output light pulses of the EDFA. It should be emphasized that our attention should be focused in the region where the pulse energy is concentrated, from -15 to $15 \mathrm{ps}$, being the rest of the phase profile meaningless. For comparison purposes, the pulse $f_{0}(t)$ at the output of the EDFA can be approximately fitted in both, modulus and phase, through a linearly chirped secant hyperbolic profile $f(t)=\operatorname{sech}\left(t / T_{0}\right) \times$ $\exp \left(-j C t^{2} / 2 T_{0}^{2}\right)$, with $T_{0}=8.51$ ps and $C=-63$, see in Fig. 8a, b the curves labeled as "sech" and "linear chirp," respectively. Essentially, the same results were obtained for the recovered intensity and phase profiles at the output of the $\operatorname{EDFA} f_{0}(t)$, when the same pre-stretching dispersion line length was used-i.e., $L_{0}=5 \mathrm{~m}$, but with a phase recovery dispersion line length of $L_{1}=4.7 \mathrm{~m}$.

When an initially up-chirped light pulse propagates under an anomalous dispersive regime, the dispersioninduced chirp is in opposite direction to that of the initial chirp. As a result, the net chirp is reduced, leading to pulse narrowing [21]. The minimum pulse width $\Delta t_{m}$ occurs at the optical fiber length $L_{m}$ in which the two chirps cancel each other. When the propagation distance is increased further, the dispersion-induced chirp starts to dominate over the initial chirp, and the pulse begins to broaden again. With the purpose to corroborate the up-chirp detected in the output light pulses of the EDFA, another set of experiments was performed by propagating this output light pulses through a dispersive line made entirely of SMF-28 optical fiber, which is anomalous at the propagating wavelength. The maximum narrowing was detected at a propagation length $L_{m}=52.5 \mathrm{~m}$, when the measured temporal width of the intensity autocorrelation was $\Delta t_{\mathrm{AUC}}=0.35 \mathrm{ps}$. A further propagation monotonously increases the temporal width again. This behavior reasonably matches with the temporal width of the calculated 
Fig. 8 Temporal intensity and phase profiles of the output light pulses of the EDFA,

experimentally recovered by the FrT, FrT-GS, and GS phase recovery techniques, (a) and (b), respectively
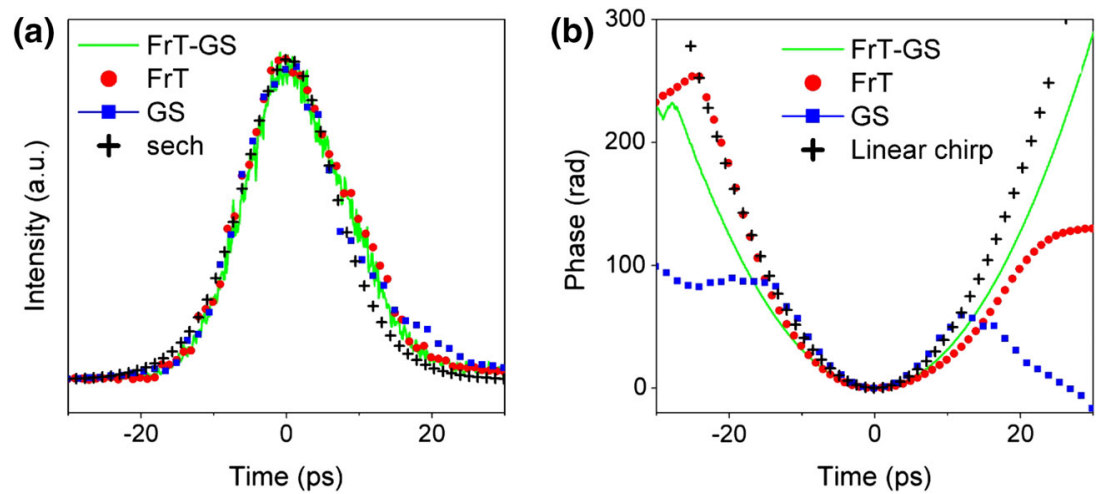

intensity autocorrelation, i.e., $\Delta t_{\mathrm{AUC}}=0.37 \mathrm{ps}$, for the theoretical pulse shown in Fig. 8, when the propagation through the same distance, i.e., $L_{m}=52.5 \mathrm{~m}$, is simulated. This further corroborates the result obtained, i.e., an approximately parabolic phase profile in the output light pulses of the EDFA, with a chirp parameter $C \cong-63$.

\section{Conclusion}

In this work, the applicability of the recently proposed FrT phase retrieval technique was extended by incorporating pulse pre-stretching, for those cases where the oscilloscope bandwidth is insufficient, or there is a physical limitation to reach the point under analysis. The proposed technique makes high-speed real-time reconstruction of light pulses possible, down to the ps regime. In the downside, a highspeed oscilloscope is necessary. As a proof of concept, the light pulses of a two-stage laser system were analyzed. The recovered temporal intensity and phase profiles were successfully compared with two indirect phase recovery techniques, both based on the GS recursive algorithm. As a result, the output light pulses of the mode-locked laser are un-chirped. On the contrary, a strong frequency up-chirp in the output light pulses of the EDFA was detected and measured.

Acknowledgments This work has been financially supported by the Ministerio de Economía y Competitividad (Ref. TEC2013-46643-C21-R) and the Generalitat Valenciana of Spain (Ref. PROMETEOII/ 2014/072 and GV/2012/121). C. Cuadrado-Laborde acknowledges the financial support from the Programa de Investigadores Invitados de la Universidad de Valencia (Spain).

\section{References}

1. T.E. Gureyev, A. Pogany, D.M. Paganin, S.W. Wilkins, Linear algorithms for phase retrieval in the Fresnel region. Opt. Commun. 231, 53-70 (2004)
2. R.W. Gerchberg, W.O. Saxton, A practical algorithm for the determination of phase from image and diffraction plane pictures. Optik 35, 237-246 (1972)

3. J. Fienup, Phase retrieval algorithms: a comparison. Appl. Opt. 21, 2758-2769 (1982)

4. M.R. Teague, Deterministic phase retrieval: a Green function solution. J. Opt. Soc. Am. 73, 1434-1441 (1983)

5. N. Streibl, Phase imaging by the transport equation of intensity. Opt. Commun. 49, 6-10 (1984)

6. K. Ichikawa, A.W. Lohmann, M. Takeda, Phase retrieval based on the irradiance transport equation and the Fourier transport method: experiments. Appl. Opt. 27, 3433-3436 (1988)

7. F. Li, Y. Park, J. Azaña, Complete temporal pulse characterization based on phase reconstruction using optical ultrafast differentiation (PROUD). Opt. Lett. 32, 3364-3366 (2007)

8. M.H. Asghari, J. Azaña, Self-referenced temporal phase reconstruction from intensity measurements using causality arguments in linear optical filters. Opt. Lett. 37, 3582-3584 (2012)

9. M.H. Asghari, B. Jalali, Stereopsis-inspired time-stretched amplified real-time spectrometer (STARS). IEEE Photonics J. 4, 1693-1701 (2012)

10. C. Cuadrado-Laborde, A. Carrascosa, P. Pérez-Millán, A. Díez, J. Cruz, M. Andres, Phase recovery by using optical fiber dispersion. Opt. Lett. 39, 598-601 (2014)

11. K. Goda, B. Jalali, Dispersive Fourier transformation for fast continuous single-shot measurements. Nat. Photonics 7, 102-112 (2013)

12. J.-H. Chung, A.M. Weiner, Ambiguity of ultrashort pulse shapes retrieved from the intensity autocorrelation and power spectrum. IEEE J. Sel. Top. Quant. Electron. 7, 656-666 (2001)

13. M.J. Bastiaans, K.B. Wolf, Phase reconstruction from intensity measurements in linear systems. J. Opt. Soc. Am. A 20, 1046-1049 (2003)

14. C. Dorrer, Characterization of nonlinear phase shifts by use of the temporal transport-of-intensity equation. Opt. Lett. 30, 32373239 (2005)

15. C. Dorrer, I. Kang, Complete temporal characterization of short optical pulses by simplified chronocyclic tomography. Opt. Lett. 28, 1481-1483 (2003)

16. D.R. Solli, S. Gupta, B. Jalali, Optical phase recovery in the dispersive Fourier transform. Appl. Phys. Lett. 95, 231108 (2009)

17. M. Muriel, J. Azaña, A. Carballar, Real-time Fourier transformer based on fiber gratings. Opt. Lett. 24, 1-3 (1999)

18. F. Li, Y. Park, J. Azaña, Linear characterization of optical pulses with durations ranging from the picosecond to the nanosecond regime using ultrafast photonic differentiation. IEEE J. Lightwave Technol. 27, 4623-4633 (2009) 
19. I. Kang, C. Dorrer, Highly sensitive differential tomographic technique for real-time ultrashort pulse characterization. Opt. Lett. 30, 1545-1547 (2005)

20. F. Li, Y. Park, J. Azaña, Linear characterization of optical pulses with durations ranging from the picosecond to the nanosecond regime using ultrafast photonic differentiation. J. Light. Technol. 27, 4623-4633 (2009)

21. G.P. Agrawal, Nonlinear Fiber Optics (Academic, San Diego, CA, 2001) 